\title{
VARIATIONAL IMAGE INPAINTING
}

\author{
Tony F. Chan * \\ Department of Mathematics, UCLA \\ Los Angeles, CA 90095, USA \\ chan@math.ucla.edu \\ Jianhong Shen ${ }^{\dagger}$ \\ School of Mathematics \\ University of Minnesota \\ Minneapolis, MN 55455, USA \\ jhshen@math.umn.edu
}

\begin{abstract}
Inpainting is an image interpolation problem, with broad applications in image and vision analysis. This paper presents our recent efforts in developing inpainting models based on the Bayesian and variational principles. We discuss several geometric image models, their role in the construction of variational inpainting models, and the associated Euler-Lagrange PDEs and their numerical computation.
\end{abstract}

Keywords: Inpainting, interpolation, Bayesian, curve model, image model, Euclidean invariance, elastica, bounded variation, Mumford-Shah, curvature, $\Gamma$-convergence, numerical PDE.

\section{Introduction}

The word "inpainting" is an artistic synonym for "image interpolation," as frequently used among museum restoration artists, who manually remove cracks from degraded ancient paintings by following as faithfully as possible the original intention of their creators [EM76, Wal85]. A mathematical illustration is depicted in Figure 1.

${ }^{*}$ Research is supported by grants from NSF under grant number DMS-9626755 and from ONR under N00014-96-1-0277.

† Research is supported by NSF under DMS-0202565. 
As fine art museums go digital, all real paintings are scanned into computers. No doubt, digital inpainting provides the safest way to restore those degraded ancient paintings, simply by trying computer codes and softwares on the digital copies. Unlike the manual inpainting process which applies directly to the original, digital inpainting brings tremendous freedom in making errors or improving results progressively, with no risk of destroying the original precious painting on the canvas, which is often unique in the entire world.

But the application of digital inpainting goes far beyond on-line art museums. In on-line real estate business, for example, a potential customer may ask: I do not like the palm tree and bush in the front yard; what will the house look like without them? If the tree and bush are too close to the house, it is impossible to capture by ordinary cameras an unblocked overview of the entire house. However, treating the tree and bush as an inpainting domain, it is very hopeful that a (clever) inpainting scheme can get rid of them in a realistic fashion.

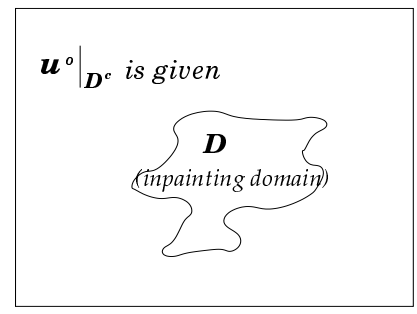

Figure 1. For a typical inpainting problem, the image is missing on an inpainting domain $D$, and the available part $\left.u^{0}\right|_{D^{c}}$ is often noisy. $D$ can be disconnected.

Ever since the original work of Bertalmio et al. [BSCB00], digital inpainting has found wide applications in image processing, vision analysis, and the movie industry. Recent examples include: automatic scratch removal in digital photos and old films [BSCB00, CS01a], text erasing $\left[\mathrm{BBC}^{+} 01\right.$, BSCB00, CS01a, CS01c], special effects such as object disappearance and wire removal for movie production [BSCB00, CS01c], disocclusion [MM98], zooming and super-resolution $\left[\mathrm{BBC}^{+} 01\right.$, CS01a, TAYW01], lossy perceptual image coding [CS01a], and removal of the laser dazzling effect [CCBT01], and so on. On the other hand, in the engineering literature, there also have been many earlier works closely related to inpainting, which include image interpolation [AKR97, KMFR95a, KMFR95b], image replacement [IP97, WL00], and error concealment [JCL94, KS93] in the communication technology.

As scattered as the applications are, the methods for inpainting related problems have also been very diversified, ranging from nonlinear filtering method, wavelets and spectral method, and statistical method (especially for textures), etc. 
The most recent approach to non-texture inpainting is based on the PDE method and Calculus of Variations, and can be classified into two categories. The first class is based on the simulation of micro-inpainting mechanisms. It includes the axiomatic approach of Caselles, Morel and Sbert [CMS98], the transport process [BSCB00] (the first high-order PDE model), the diffusion process [CS01c], and their combination [BBS01, CS01b]. The second category includes all variational models simulating the unique macro-inpainting mechanism: "best guess," or the Bayesian framework [GG84, KR96, Mum94b]. The latter includes the total variation model [CS01d, CS01a, RO94, ROF92], the functionalized elastica model [CKS01, MM98], the value-and-direction joint model $\left[\mathrm{BBC}^{+} 01\right]$, the active contour model based on Mumford and Shah's segmentation [TAYW01], and the inpainting scheme based on the MumfordShah-Euler image model [ES02].

The current paper surveys and summarizes this latter category. The main goal is to develop a systematic approach and mathematical foundation for all these previously scattered works, so that the survey can serve as a fresh starting point, rather than a concluding chapter, for further research on this challenging topic.

The philosophy behind Bayesian inpainting is quite simple and intuitive (see Figure 1): the way we human inpainters inpaint an incomplete picture mostly relies on two factors - how we read the existing part of the picture $\left.u^{0}\right|_{\Omega \backslash D}$ (i.e. data model), and what class of images we believe the original good picture $u$ belongs to (i.e. image prior model). (For example, if it is known that we are inpainting an image of a kitchen with tomatoes, peppers, and apples, we have the a priori preference of smooth shapes and the green and red colors.) In the Bayesian language, a balanced optimal guess is to maximize the posterior probability $\operatorname{Prob}\left(u \mid u^{0}\right)$ (MAP) given by

$$
\operatorname{Prob}\left(u \mid u^{0}\right)=\frac{\operatorname{Prob}\left(u^{0} \mid u\right) \cdot \operatorname{Prob}(u)}{\operatorname{Prob}\left(u^{0}\right)} .
$$

Once an image $u^{0}$ is given, the denominator is a fixed constant. Thus we are to maximize the product of the data probability and the image probability.

For inpainting, the data model is usually simple as illustrated in Figure 1: the available part $\left.u^{0}\right|_{\Omega \backslash D}$ is the restriction of the original good image $u$ on $\Omega \backslash D$, polluted independently by white noise $n$, i.e.

$$
\left.u^{0}\right|_{\Omega \backslash D}=\left.u\right|_{\Omega \backslash D}+n \text {. }
$$

On the other hand, since there is no data available on the inpainting domain $D$, the task of reconstructing the image on $D$ solely falls on the image model. This makes a good image model more crucial for inpainting than for any other classical restoration problems such as denoising, deblurring, and segmentation [CSO1a, ES02]. 
Image models can be learned from image data banks based on filtering, parametric or non-parametric estimation, and the entropy method (See Zhu, Wu and Mumford [ZM97, ZWM97] for examples). Such statistical approach is especially important for inpainting or synthesizing images with rich textures [IP97, WL00].

On the other hand, in most inpainting problems, the inpainting domain often "erases" some perceptually important geometric information of the image, edges, for example. To reconstruct geometry, it is necessary that the image model well resolves the geometry a priori. Most conventional probabilistic models lack such feature. Fortunately, "energy" forms do exist in the literature, which have been explicitly motivated by geometry. Well-known examples include the TV (total variation) model of Rudin, Osher and Fatemi [ROF92] and the object-edge model of Mumford and Shah [MS89]. The link between probabilistic image models and such geometric image models, as Mumford pointed out [Mum94b], is formally made through Gibbs' formula in statistical mechanics [Gib02]:

$$
\operatorname{Prob}(u)=\frac{1}{Z} \exp (-\beta E[u])
$$

where $E[u]$ is the energy of $u$ (e.g., the total variation of $u$ ), $\beta$ denotes the inverse absolute temperature, and $Z$ the partition function. (Working with energy also frees one from laboring on the definability of the partition function $Z$, which is generally a highly non-trivial mathematical issue.) The Bayesian formula (1) is re-expressed in the energy form by

$$
E\left[u \mid u^{0}\right]=E\left[u^{0} \mid u\right]+E[u]+\text { const. }
$$

where the constant can be dropped safely as far as energy minimization is concerned.

The organization goes as follows. Section 2 starts with an axiomatic approach for curve models, which, to our best knowledge, is new. The latter half of the section explains two approaches for constructing geometric image models from curve models:

(i) through direct functionalization based on the level-sets; and

(ii) by having a curve model embedded as an edge model in the object-edge primitive image model.

These approaches unify the four geometric image models appearing in the recent inpainting literature:

(1) the TV image model of Rudin, Osher and Fatemi [ROF92, RO94], first applied to inpainting modeling by Chan and Shen [CSO1a]; 
(2) the functionalized elastica image model as proposed and studied by Masnou and Morel [MM98], and Chan, Kang, and Shen [CKS01];

(3) the Mumford-Shah image model [MS89] applied to inpainting by Tsai, Yezzi, and Willsky [TAYW01], Chan and Shen [CS01a], and Esedoglu and Shen [ES02]; and

(4) the Mumford-Shah-Euler image model designed for image inpainting by Esedoglu and Shen [ES02].

Section 3 explains all the recent inpainting schemes based on these geometric image models. We discuss the associated Euler-Lagrange PDE's, their geometric meaning, and robust ways of numerical implementation. Digital examples are given for each inpainting model. Conclusion and open problems are written into Section 4.

Throughout the paper, $\Omega$ denotes the entire image domain, $D$ the missing inpainting domain, $u^{0}$ the available part of the image on $\Omega \backslash D$, and $u$ the targeted inpainting restoration. The standardized symbols $\nabla, \nabla \cdot$ and $\Delta$ represent the gradient, divergence, and Laplacian operators separately. For any multi-variable function or functional $F(X, Y)$, the symbol $F(X \mid Y)$ still means $F(X, Y)$, but emphasizing that $Y$ is fixed as known. This is to imitate the symbol of conditional probability or expectation appearing in the Bayesian formula (but without probabilistic normalization).

\section{Geometric Image Models}

\subsection{Curve models}

Geometry plays a crucial role in visual perception and image understanding, including classification and pattern recognition. The most important geometry for image analysis is hidden in edges. From David Marr's classical work on primal sketch [MH80] to David Donoho's geometric wavelets analysis [Don00], edges always stay in the heart of many issues: image coding and compression, image restoration, segmentation and tracking, just to name a few.

Therefore it is of fundamental significance to understand how to mathematically model edges and curves.

From the Bayesian point of view, this is to establish a probability distribution Prob $(\Gamma)$ over "all" curves. An instant example coming to mind is the Brownian motion and its Wiener measure [KS97]. The problem is that Brownian paths are parameterized curves (by "time") and are even almost surely no-where differentiable. For image analysis, however, edges are intrinsic (1D) manifolds and their regularity is an important visual cue.

According to the previously stated Gibbs' formulation, we are to look for a suitable energy form $E[\Gamma]$. It is always convenient to first start with its digital version. 
In digital image processing, Freeman's Chain Coding [Fre61] is a popular data structure for representing object borders and edges. The underlying idea is to represent a 1-D curve $\Gamma$ by a chain of ordered sample points

$$
\boldsymbol{x}_{0}, \boldsymbol{x}_{1}, \cdots, \boldsymbol{x}_{N},
$$

dense enough to ensure reasonable approximation. Working directly with such chains of finite length, we need to define appropriate energy forms

$$
E\left[\boldsymbol{x}_{0}, \boldsymbol{x}_{1}, \cdots, \boldsymbol{x}_{N}\right] .
$$

To the best of our knowledge, the following axiomatic approach is new in the literature. We shall naturally construct two of the most useful planer curve models: the length energy and Euler's elastica energy.

Axiom 1. Euclidean invariance.

Let $Q \in O(2)$ (conventionally called a rotation, though including all reflections), and $c \in R^{2}$ an arbitrary point. Euclidean invariance consists of two parts: the rotational invariance

$$
E\left[Q \boldsymbol{x}_{0}, Q \boldsymbol{x}_{1}, \cdots, Q \boldsymbol{x}_{N}\right]=E\left[\boldsymbol{x}_{0}, \boldsymbol{x}_{1}, \cdots, \boldsymbol{x}_{N}\right],
$$

and the translation invariance

$$
E\left[\boldsymbol{x}_{0}+\boldsymbol{c}, \boldsymbol{x}_{1}+\boldsymbol{c}, \cdots, \boldsymbol{x}_{N}+\boldsymbol{c}\right]=E\left[\boldsymbol{x}_{0}, \boldsymbol{x}_{1}, \cdots, \boldsymbol{x}_{N}\right] .
$$

Axiom 2. Reversal invariance.

It requires that

$$
E\left[\boldsymbol{x}_{0}, \cdots, \boldsymbol{x}_{N}\right]=E\left[\boldsymbol{x}_{N}, \cdots, \boldsymbol{x}_{0}\right],
$$

which means that the energy does not depend on the orientation of the curve.

Axiom 3. $p$-point accumulation $(p=2,3, \cdots)$.

This is fundamentally a rule on locality. A $p$-point accumulative energy satisfies the accumulation law:

$$
E\left[\boldsymbol{x}_{0}, \cdots, \boldsymbol{x}_{n}, \boldsymbol{x}_{n+1}\right]=E\left[\boldsymbol{x}_{0}, \cdots, \boldsymbol{x}_{n}\right]+E\left[\boldsymbol{x}_{n-p+2}, \cdots, \boldsymbol{x}_{n+1}\right],
$$

for all $n \geq p-2$. Through cascade, we easily establish that 
Proposition 1 Suppose $E$ is p-point accumulative. Then for any $N \geq p-1$,

$$
E\left[\boldsymbol{x}_{0}, \cdots, \boldsymbol{x}_{N}\right]=\sum_{n=0}^{N-p+1} E\left[\boldsymbol{x}_{n}, \cdots, \boldsymbol{x}_{n+p-1}\right]
$$

Thus, for example, a 2-point accumulative energy must be in the form of

$$
E\left[\boldsymbol{x}_{0}, \cdots, \boldsymbol{x}_{N}\right]=E\left[\boldsymbol{x}_{0}, \boldsymbol{x}_{1}\right]+E\left[\boldsymbol{x}_{1}, \boldsymbol{x}_{2}\right]+\cdots+E\left[\boldsymbol{x}_{N-1}, \boldsymbol{x}_{N}\right] ;
$$

and a 3-point accumulative energy satisfies

$$
E\left[\boldsymbol{x}_{0}, \cdots, \boldsymbol{x}_{N}\right]=E\left[\boldsymbol{x}_{0}, \boldsymbol{x}_{1}, \boldsymbol{x}_{2}\right]+E\left[\boldsymbol{x}_{1}, \boldsymbol{x}_{2}, \boldsymbol{x}_{3}\right]+\cdots+E\left[\boldsymbol{x}_{N-2}, \boldsymbol{x}_{N-1}, \boldsymbol{x}_{N}\right] .
$$

Generally, a $p$-point accumulative energy $E$ is completely determined by its fundamental form

$$
E\left[\boldsymbol{x}_{0}, \cdots, \boldsymbol{x}_{p-1}\right]
$$

In what follows, we study the cases of $p=2$ and $p=3$.

\section{2-point accumulative energy and the length .}

Proposition 2 A Euclidean invariant 2-point accumulative energy must be in the form of

$$
E\left[\boldsymbol{x}_{0}, \cdots, \boldsymbol{x}_{N}\right]=\sum_{n=0}^{N-1} f\left(\left|\boldsymbol{x}_{n+1}-\boldsymbol{x}_{n}\right|\right),
$$

for some non-negative function $f(s)$.

Proof. We only need to show that

$$
E\left[\boldsymbol{x}_{0}, \boldsymbol{x}_{1}\right]=f\left(\left|\boldsymbol{x}_{1}-\boldsymbol{x}_{0}\right|\right) .
$$

Translation invariance leads to

$$
E\left[\boldsymbol{x}_{0}, \boldsymbol{x}_{1}\right]=E\left[0, \boldsymbol{x}_{1}-\boldsymbol{x}_{0}\right]=F\left(\boldsymbol{x}_{1}-\boldsymbol{x}_{0}\right),
$$

with $F(\boldsymbol{x})=E[0, \boldsymbol{x}]$. Then rotational invariance further implies that

$$
F(Q \boldsymbol{x}) \equiv F(\boldsymbol{x}), \quad Q \in O(2), \boldsymbol{x} \in R^{2} .
$$

Thus if we define $f(s)=F((s, 0))$, then $F(\boldsymbol{x})=f(|\boldsymbol{x}|)$.

If in addition, we impose

Axiom 4. Linear additivity:

For any $\alpha \in(0,1)$, and $\boldsymbol{x}_{1}=\alpha \boldsymbol{x}_{0}+(1-\alpha) \boldsymbol{x}_{2}$,

$$
E\left[\boldsymbol{x}_{0}, \boldsymbol{x}_{2}\right]=E\left[\boldsymbol{x}_{0}, \boldsymbol{x}_{1}\right]+E\left[\boldsymbol{x}_{1}, \boldsymbol{x}_{2}\right] .
$$


Then it is easy to show that the energy is unique up to a multiplicative constant.

Theorem 1 A Euclidean invariant 2-point accumulative energy $E$ with linear additivity must be the length energy, i.e.,

$$
E\left[\boldsymbol{x}_{0}, \cdots, \boldsymbol{x}_{N}\right]=c \sum_{n=0}^{N-1}\left|\boldsymbol{x}_{n}-\boldsymbol{x}_{n+1}\right|,
$$

for some fixed positive constant $c$.

For a summable curve $\Gamma$, as $N \rightarrow \infty$, and the sampling size

$$
\max _{0 \leq n \leq N-1}\left|\boldsymbol{x}_{n}-\boldsymbol{x}_{n+1}\right|
$$

tends to zero, such digital energy converges to the length.

\section{3-point accumulative energy and the curvature.}

To determine the fundamental form $E\left[\boldsymbol{x}_{0}, \boldsymbol{x}_{1}, \boldsymbol{x}_{2}\right]$, first recall Frobenius' classical theorem [COS ${ }^{+}$98]. The three points $\boldsymbol{x}_{0}, \boldsymbol{x}_{1}, \boldsymbol{x}_{2}$ live in $R^{6}=R^{2} \times$ $R^{2} \times R^{2}$, and the dimension of a Euclidean orbit is 3: 1 from the rotation group, and 2 from the translation group. Therefore, Frobenius' theorem applied to the Euclidean invariance gives

Proposition 3 One can find exactly three independent joint invariants: $I_{1}, I_{2}$, and $I_{3}$, such that $E\left[\boldsymbol{x}_{0}, \boldsymbol{x}_{1}, \boldsymbol{x}_{2}\right]$ is a function of them.

Define

$$
a=\left|\boldsymbol{x}_{1}-\boldsymbol{x}_{0}\right|, \quad b=\left|\boldsymbol{x}_{2}-\boldsymbol{x}_{1}\right|, \quad c=\left|\boldsymbol{x}_{2}-\boldsymbol{x}_{0}\right| .
$$

Then the ordered triple $(a, b, c)$ is apparently Euclidean invariant, and two chains $\left[\boldsymbol{x}_{0}, \boldsymbol{x}_{1}, \boldsymbol{x}_{2}\right]$ and $\left[\boldsymbol{y}_{0}, \boldsymbol{y}_{1}, \boldsymbol{y}_{2}\right]$ are Euclidean congruent if and only if they share the same $(a, b, c)$. Thus there must exist a non-negative function $F(a, b, c)$ such that

$$
E\left[\boldsymbol{x}_{0}, \boldsymbol{x}_{1}, \boldsymbol{x}_{2}\right]=F(a, b, c) .
$$

Define the two elementary symmetric functions of $a$ and $b$ :

$$
A_{1}=\frac{a+b}{2} \text { and } B_{1}=a b .
$$

The reversal invariance implies the symmetry of $F$ with respect to $a$ and $b$. Thus $E$ has to be a function of $A_{1}, B_{1}$, and $c$ :

$$
E\left[\boldsymbol{x}_{0}, \boldsymbol{x}_{1}, \boldsymbol{x}_{2}\right]=f\left(A_{1}, B_{1}, c\right) .
$$


Let $s$ denote the half perimeter of the triangle $\left(\boldsymbol{x}_{1}, \boldsymbol{x}_{2}, \boldsymbol{x}_{3}\right)$ :

$$
s=A_{1}+\frac{c}{2}
$$

and $\Delta$ its area:

$$
\Delta=\sqrt{s(s-a)(s-b)(s-c)}=\sqrt{s(s-c)\left(s^{2}-2 A_{1} s+B_{1}\right)} .
$$

Then we can define the digital curvature at $\boldsymbol{x}_{1}\left[\mathrm{COS}^{+} 98\right.$, COT96, Bou00] by

$$
\kappa_{1}=4 \frac{\Delta}{B_{1} c}=\frac{\sin \theta_{1}}{c / 2}
$$

where $\theta_{1}$ is the angle facing the side $\left[\boldsymbol{x}_{0}, \boldsymbol{x}_{2}\right]$. It is shown by Calabi, Olver, and Tannenbaum [COT96] that for a generic smooth curve and a fixed point $\boldsymbol{x}_{1}$ on it, as $a, b \rightarrow 0$,

$$
\kappa_{1}=\kappa\left(\boldsymbol{x}_{1}\right)+O(|b-a|)+O\left(a^{2}+b^{2}\right),
$$

where $\kappa\left(\boldsymbol{x}_{1}\right)$ is the curvature at $\boldsymbol{x}_{1}$.

Now it is easy to see that $\kappa_{1}, A_{1}, B_{1}$ is a complete set of joint invariants for both the Euclidean and reversal invariances, and

$$
E\left[\boldsymbol{x}_{0}, \boldsymbol{x}_{1}, \boldsymbol{x}_{2}\right]=g\left(\kappa_{1}, A_{1}, B_{1}\right) .
$$

Therefore, we have proved

Theorem 2 A 3-point accumulative energy $E$ with both Euclidean and reversal invariances must be in the form of

$$
E\left[\boldsymbol{x}_{0}, \cdots, \boldsymbol{x}_{N}\right]=\sum_{n=1}^{N-1} g\left(\kappa_{n}, A_{n}, B_{n}\right) .
$$

Further notice that, as the sampling size $a, b=O(h), h \rightarrow 0$ at a fixed point $\boldsymbol{x}_{1} \in \Gamma$,

$$
\kappa_{1}=O(1), \quad A_{1}=O(h), \quad B_{1}=O\left(h^{2}\right) .
$$

Applying Taylor expansion to $g$ for the infinitesimals $A_{1}$ and $B_{1}$ (assuming that $g$ is smooth), we have

$$
g\left(\kappa_{1}, A_{1}, B_{1}\right)=g_{1}\left(\kappa_{1}\right) A_{1}+g_{2}\left(\kappa_{1}\right) B_{1}+\cdots,
$$

for some functions $g_{1}, g_{2}, \cdots$. In the linear integration theory, by neglecting all high order $(\geq 2)$ infinitesimals, we end up with

$$
g\left(\kappa_{1}, A_{1}, B_{1}\right)=g_{1}\left(\kappa_{1}\right) A_{1} .
$$


Therefore we have derived,

Corollary 1 Following Theorem 2, in addition, suppose that for any smooth summable simple curve $\Gamma$, and its Chain Coding approximation $\left[\boldsymbol{x}_{0}, \boldsymbol{x}_{1}, \cdots \boldsymbol{x}_{N}\right]$ with the size

$$
h=\max _{0 \leq n \leq N-1}\left|\boldsymbol{x}_{n}-\boldsymbol{x}_{n+1}\right|
$$

tending to zero, $E\left[\boldsymbol{x}_{0}, \cdots \boldsymbol{x}_{N}\right]$ converges. Then as far as the limit is concerned, there is only one class of such energy, which is given by

$$
E\left[\boldsymbol{x}_{0}, \cdots, \boldsymbol{x}_{N}\right]=\sum_{n=1}^{N-1} f\left(\kappa_{n}\right) A_{n} .
$$

As $h \rightarrow 0$, it converges to

$$
E[\Gamma]=\int_{\Gamma} f(\kappa) d s
$$

where $d s$ is the length element.

For instance, if we take $f(\kappa)=\alpha+\beta \kappa^{2}$ for two fixed weights $\alpha$ and $\beta$, the resulting energy is called the elastica energy [Mum94a], which was first studied by Euler in modeling the shape of a torsion free thin rod in 1744. If $\beta=0$, the elastica energy degenerates to the length energy.

\subsection{Image models via functionalizing curve models}

Once a curve model $E[\Gamma]$ is established, it can be "lifted" to an image model by direct functionalization and the level-set approach.

Let $u(x)$ be an image defined on a domain $\Omega \subset R^{2}$. For the moment assume that $u$ is smooth so that almost surely for each gray value $\lambda$, the level-set

$$
\Gamma_{\lambda}=\{x \in \Omega: u(x)=\lambda\}
$$

is a smooth 1-D manifold. Let $w(\lambda)$ be an appropriate non-negative weight function. Then based on a given curve model $E[\Gamma]$, we can construct an image model:

$$
E[u]=\int_{-\infty}^{\infty} E\left[\Gamma_{\lambda}\right] w(\lambda) d \lambda .
$$

Conventionally $w(\lambda)$ is set to 1 to reflect human perceptual sensitivity. Suppose we have a bundle of level-sets whose gray values are concentrated over $[\lambda, \lambda+\Delta \lambda]$. If $\Delta \lambda$ is small, then the image appears smooth over the region made of these level-sets, and is thus less sensitive to perception. The energy 
assigned to such bundles should be small accordingly. On the other hand, if $\Delta \lambda$ is large, for example in the situation when the bundle contains a sharp edge, then the level-sets carry important visual information and the associated energy should be large. Therefore, the Lebesgue measure $d \lambda$ is already perceptually motivated and $w(\lambda)$ can be set to 1 , which we shall assume in the following.

Suppose we take the length energy in Theorem 1 as the curve model, then the resulting image model

$$
E[u]=\int_{-\infty}^{\infty} \operatorname{length}\left(\Gamma_{\lambda}\right) d \lambda
$$

is exactly Rudin-Osher-Fatemi's TV model [ROF92, RO94]:

$$
E[u]=\int_{\Omega}|\nabla u| d x .
$$

This is because for a smooth image $u$, along any level-set $\Gamma_{\lambda}$,

$$
d \lambda=|\nabla u| d \sigma, \quad \text { length }\left(\Gamma_{\lambda}\right)=\int_{\Gamma_{\lambda}} d s
$$

with $d s$ and $d \sigma$ denoting the arc lengthes of the level-sets and gradient flows, which are orthogonal to each other, and thus

$$
d s d \sigma=d x
$$

is the area element. Therefore,

$$
E[u]=\int_{-\infty}^{\infty} \int_{\Gamma_{\lambda}}|\nabla u| d \sigma d s=\int_{\Omega}|\nabla u| d x .
$$

The above derivation is in a formal level and can be rigorously established based on the theory of BV functions [Giu84], where the length of a level-set is replaced by the perimeter of its associated region, the Sobolev gradient norm by the TV radon measure. Then the lifting process is exactly the famous coarea formula.

Similarly, suppose we take the curvature curve model in Corollary 1, then the lifted image model becomes

$$
E[u]=\int_{\Omega} f\left(\left|\nabla \cdot\left[\frac{\nabla u}{|\nabla u|}\right]\right|\right)|\nabla u| d x .
$$

Especially, if $f(s)=\alpha+\beta s^{2}$, it is called the elastica image model [CKS01, MM98]. 


\subsection{Image models with embedded edge models}

The second approach to construct image models from curve models is based on the object-edge primary model, as proposed by Mumford-Shah [MS89]. In such image models, the curve model is embedded to weigh the energy from the edges, i.e., abrupt jumps in images.

For example, the classical Mumford-Shah image model employs the length curve model:

$$
E[u, \Gamma]=\int_{\Omega \backslash \Gamma}|\nabla u|^{2} d x+\alpha \text { length }(\Gamma) .
$$

Here $\Gamma$ denotes edge collection. Unlike the TV image model, once the singular set $\Gamma$ is singled out, for the rest of the image domain, Sobolev smoothness can be legally imposed.

Mumford-Shah image model has been very successful in image segmentation and denoising. For image inpainting, as Esedoglu-Shen discussed in [ES02], it is intrinsically insufficient. Therefore a new image model called MumfordShah-Euler is proposed in [ES02] based on the elastica curve model:

$$
E[u, \Gamma]=\int_{\Omega \backslash \Gamma}|\nabla u|^{2} d x+\int_{\Gamma}\left(\alpha+\beta \kappa^{2}\right) d s .
$$

We now start to discuss how to carry out inpainting based on these image models.

\section{Inpainting Models and Their PDE's}

In this section, we survey all the recent inpainting schemes based on the geometric image models mentioned above. We shall describe the PDE forms for all the variational models, and their digital realization based on the numerical PDE method. Digital examples are demonstrated for each inpainting scheme.

\subsection{The TV inpainting}

In [CS01a], we first touched on the Bayesian idea for the inpainting problem, as an alternative to the PDE approach invented by Bertalmio et al. [BSCB00] based on the transport mechanism. The image model employed in [CS01a] is the well-known Rudin-Osher-Fatemi's TV image model, as first proposed for the denoising and deblurring application [ROF92, RO94].

The TV inpainting model is to minimize the posterior energy

$$
J_{\mathrm{tv}}\left[u \mid u^{0}, D\right]=\int_{\Omega}|\nabla u| d x+\frac{\lambda}{2} \int_{\Omega \backslash D}\left(u-u^{0}\right)^{2} d x .
$$

Define

$$
\lambda_{D}(x)=\lambda \cdot 1_{\Omega \backslash D}(x) .
$$


Then the steepest descent equation for the energy is

$$
\frac{\partial u}{\partial t}=\nabla \cdot\left[\frac{\nabla u}{|\nabla u|}\right]+\lambda_{D}(x)\left(u^{0}-u\right)
$$

which is a diffusion-reaction type of nonlinear equation. To justify the drop of the boundary integral coming from the variational process, the associated boundary condition along $\partial \Omega$ is adiabatic: $\partial u / \partial \vec{\nu}=0$, where $\vec{\nu}$ denotes the normal direction of the boundary.

The diffusion is anisotropic to respect sharp edges as in the Perona-Malik diffusion [PM90] since the diffusivity coefficient $1 /|\nabla u|$ becomes small where $u$ has sharp jumps. The reaction term has $u^{0}$ as its attractor to keep the solution close to the given noisy image. But notice that on the inpainting domain $D$, the equation is a pure diffusion process, which of course originally comes from the TV image model.

In [CKS01], the existence of a minimizer of $J_{\mathrm{tv}}$ in the BV space is established based on the direct method of Calculus of Variation. The uniqueness, however, is generally not guaranteed. An example is given in [CKS01]. Nonuniqueness, from the vision point of view, reflects the uncertainty of human visual perception in certain situations, and thus should not be cursed in terms of faithful modeling.

For the digital realization of model (3), the degenerate diffusion coefficient $1 /|\nabla u|$ is always conditioned to

$$
\frac{1}{|\nabla u|_{a}}, \quad|\nabla u|_{a}=\sqrt{a^{2}+|\nabla u|^{2}}
$$

for some small positive constant $a$. From the energy point of view, it amounts to the minimization of the modified $J_{\mathrm{tv}}$ :

$$
J_{\mathrm{tv}}^{a}[u]=\int_{\Omega}|\nabla u|_{a}+\frac{\lambda}{2} \int_{\Omega \backslash D}\left(u-u^{0}\right)^{2} d x .
$$

This energy form connects image inpainting to the classical problem of nonparametric minimal surfaces [Giu84]. In fact, in the $(x, y, z)$ space, the first term of $J_{\mathrm{tv}}^{a}[u]$, up to the multiplicative constant $a$, is exactly the area of the surface

$$
z=z(x, y)=u(x, y) / a .
$$

In the case when the available part $u^{0}$ is noise-free, we have

$$
\lambda=\infty,\left.\quad z\right|_{\Omega \backslash D}=\left.z^{0}\right|_{\Omega \backslash D} .
$$

Thus we end up with the exact minimal surface problem on the inpainting domain $D$ :

$$
\min \int_{D} \sqrt{1+|\nabla z|^{2}} d x \quad \text { with } \quad z=z^{0} \text { along } \partial D
$$


Here along the boundary, $\left.z\right|_{\partial D}$ is understood as the trace from the interior. Since this Dirichlet problem might not be solvable for general inpainting domains $D$ (see [Giu84] for example), as far as inpainting is concerned, we may formulate a weaker version even for the noise-free case:

$$
\min \int_{D} \sqrt{1+|\nabla z|^{2}}+\frac{\mu}{2} \int_{\partial D}\left(z-z^{0}\right)^{2} d H_{1}
$$

where $\mu$ is a large positive weight and $d H_{1}$ the 1-dimensional Hausdorff measure of $\partial D$. Then the existence of a minimum can be easily established based on the direct method.

Compared with all other variational inpainting schemes, the TV model has the lowest complexity and easiest digital implementation. It works remarkably well for more local inpainting problems such as digital zoom-in (Figure 2) and text removal [CS01a]. But for large-scale inpainting problems, the TV inpainting model suffers from its origin in the length curve energy. One major drawback is its failure to realize the Connectivity Principle in visual perception as discussed in [CSO1c].
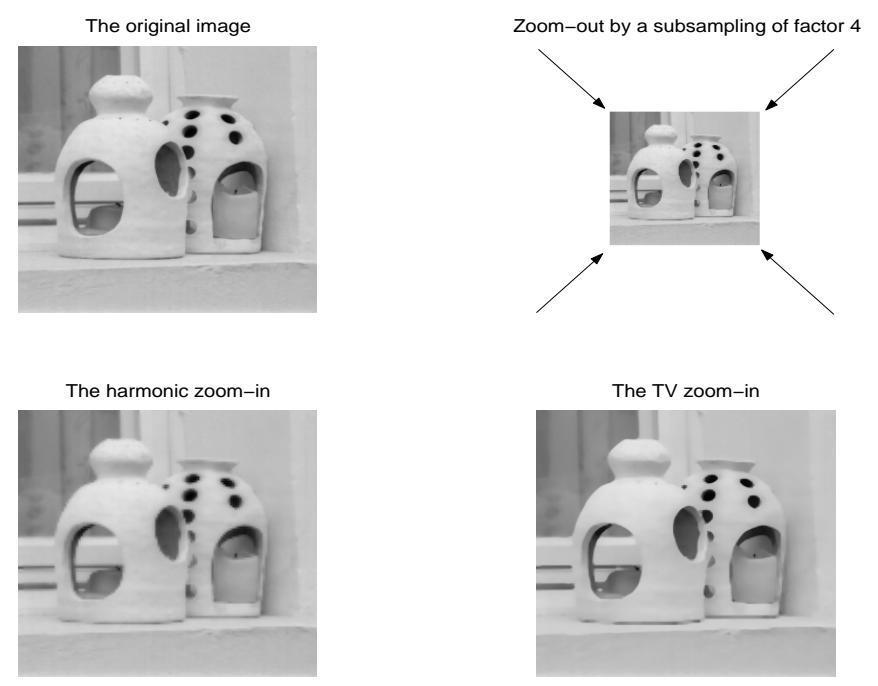

Figure 2. Digital zoom-in based on the TV inpainting scheme, as compared with that based on the harmonic inpainting scheme, i.e., that based on the Sobolev image model: $E[u]=$ $\int_{\Omega}|\nabla u|^{2} d x$. Notice that the TV gives much sharper boundary reconstruction. 

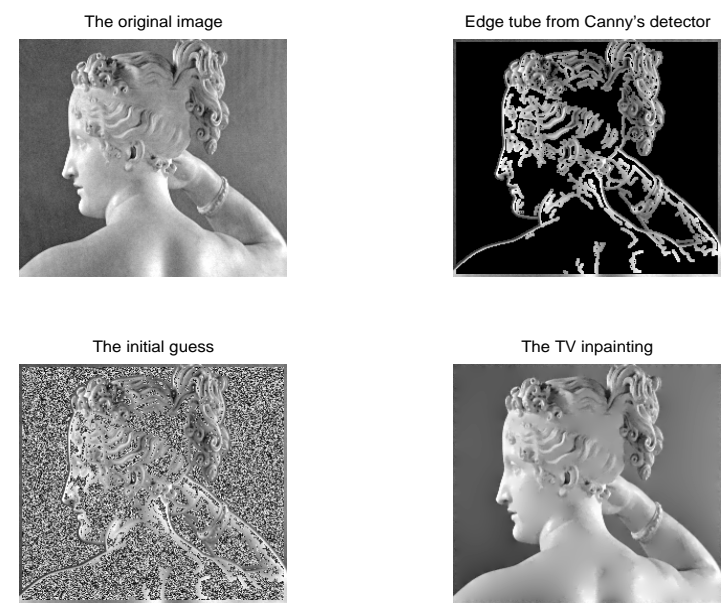

Figure 3. TV inpainting applied to the primal-sketch based image decoding.

\subsection{The elastica inpainting}

In [CKS01], Chan, Kang, and Shen proposed to improve the TV inpainting model by using the elastica image model

$$
E[u]=\int_{\Omega}\left(\alpha+\beta \kappa^{2}\right) d x, \quad \kappa=\nabla \cdot\left[\frac{\nabla u}{|\nabla u|}\right] .
$$

The elastica inpainting model is thus to minimize the posterior energy

$$
J_{\mathrm{e}}\left[u \mid u^{0}, D\right]=\int_{\Omega} \phi(\kappa) d x+\frac{\lambda}{2} \int_{\Omega \backslash D}\left(u-u^{0}\right)^{2} d x,
$$

where $\phi(s)=\alpha+\beta s^{2}$.

By Calculus of Variation, it is shown in [CKS01] that the steepest descent equation is given by

$$
\begin{aligned}
\frac{\partial u}{\partial t} & =\nabla \cdot \vec{V}+\lambda_{D}(x)\left(u^{0}-u\right), \\
\vec{V} & =\phi(\kappa) \vec{n}-\frac{\vec{t}}{|\nabla u|} \frac{\partial\left(\phi^{\prime}(\kappa)|\nabla u|\right)}{\partial \vec{t}}, .
\end{aligned}
$$

Here $\vec{n}, \vec{t}$ are the normal and tangent directions:

$$
\vec{n}=\frac{\nabla u}{|\nabla u|}, \quad \vec{t}=\vec{n}^{\perp}, \quad \frac{\partial}{\partial \vec{t}}=\vec{t} \cdot \nabla .
$$


Notice that the coupling of $\vec{t}$ and $\partial / \partial \vec{t}$ in (7) makes it safe to take any direction of $\vec{n}^{\perp}$ for $\vec{t}$. The natural boundary conditions along $\partial \Omega$ are

$$
\frac{\partial u}{\partial \vec{\nu}}=0 \quad \text { and } \quad \frac{\partial\left(\phi^{\prime}(\kappa)|\nabla u|\right)}{\partial \vec{\nu}}=0 .
$$

The vector field $\vec{V}$ is called the flux of the elastica energy. Its decomposition in the natural orthogonal frame $(\vec{n}, \vec{t})$ in (7) has significant meaning in terms of micro-inpainting mechanisms.

(i) The normal flow $\phi(k) \vec{n}$ carries the feature of an important inpainting scheme invented earlier by Chan and Shen called CDD (curvature driven diffusion) [CS01c]. CDD was discovered in looking for micro mechanisms that can realize the Connectivity Principle in visual perception [CS01c, Kan79, NMS93].

(ii) The tangential component can be written as

$$
\vec{V}_{t}=-\left(\frac{1}{|\nabla u|^{2}} \frac{\partial\left(\phi^{\prime}(\kappa)|\nabla u|\right)}{\partial \vec{t}}\right) \nabla^{\perp} u
$$

and its divergence is

$$
\nabla \cdot \vec{V}_{t}=\nabla^{\perp} u \cdot \nabla\left(\frac{-1}{|\nabla u|^{2}} \frac{\partial\left(\phi^{\prime}(\kappa)|\nabla u|\right)}{\partial \vec{t}}\right)
$$

since $\nabla^{\perp} u$ is divergence free. Define the smoothness measure

$$
L_{\phi}=\frac{-1}{|\nabla u|^{2}} \frac{\partial\left(\phi^{\prime}(\kappa)|\nabla u|\right)}{\partial \vec{t}} .
$$

Then the tangent component is in the form of the transport inpainting mechanism as originally invented by Bertalmio et al. [BSCB00].

Pure transport can lead to shocks as in traffic models, while pure curvature driven diffusion (CDD) is only motivated by the Connectivity Principle in vision research and lacks theoretical support. The elastica inpainting PDE (6) combines their strength and also offers a theoretical framework.

For the numerical realization of the model, we mention the following aspects. More detail can be found in [CKS01]. Two digital examples are illustrated in Figure 4.

(a) To accelerate the convergence of the steepest descent marching (6) toward its equilibrium solution, one can adopt the Marquina-Osher method [MO99] by adding a non-negative "time correcting factor" $T(u,|\nabla u|)$ :

$$
\frac{\partial u}{\partial t}=T \cdot\left(\nabla \cdot \vec{V}+\lambda_{D}(x)\left(u^{0}-u\right)\right) .
$$


For instance, take $T=|\nabla u|$. As shown in [MO99], such simple technique can substantially improve the numerical marching size and speed up the convergence.

(b) As in the TV inpainting model, for the computation of $\kappa$ and $\vec{V}, 1 /|\nabla u|$ is always conditioned to $1 /|\nabla u|_{a}$ to avoid a zero denominator.

(c) To more efficiently denoise and propagate sharp edges, classical numerical techniques from computational fluid dynamics (CFD) can be very useful, including those originally designed for capturing shocks. Techniques adopted in [CKS01] are the upwind scheme and the min-mod scheme [OR90].
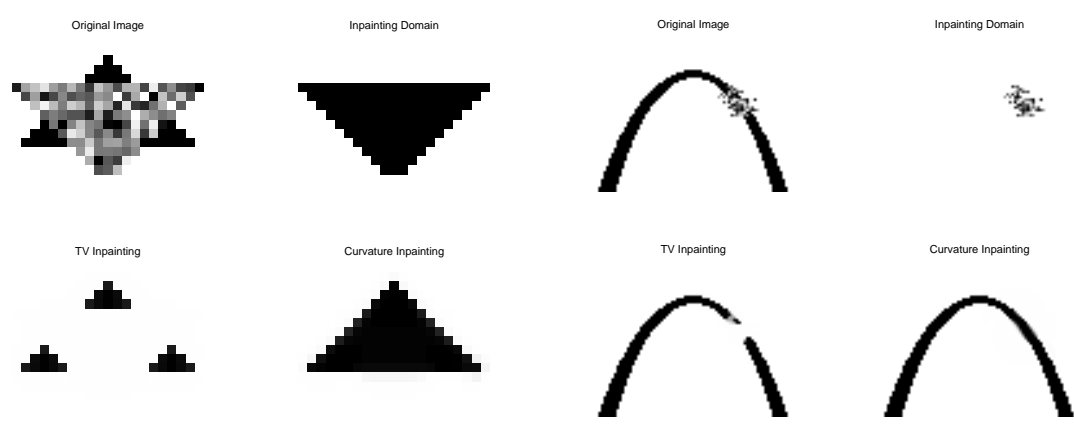

Figure 4. Two examples of elastica inpainting, as compared with TV inpainting. In the case of large aspect ratios [CS01c], the TV inpainting model fails to comply to the Connectivity Principle.

\subsection{Inpainting via Mumford-Shah image model}

The idea of applying the Mumford-Shah image model to inpainting and image interpolation first appeared in Tsai, Yezzi, and Willsky [TAYW01], and Chan and Shen [CSO1a], and has been recently studied again by Esedoglu and Shen [ES02] based on the $\Gamma$-convergence theory.

The model is to minimize the posterior energy

$$
J_{\mathrm{ms}}\left[u, \Gamma \mid u^{0}, D\right]=\frac{\gamma}{2} \int_{\Omega \backslash \Gamma}|\nabla u|^{2} d x+\alpha \text { length }(\Gamma)+\frac{\lambda}{2} \int_{\Omega \backslash D}\left(u-u^{0}\right)^{2} d x
$$

where $\gamma, \alpha$, and $\lambda$ are positive weights. Notice that if $D$ is empty, i.e. there is no spatially missing domain, then the model is exactly the classical MumfordShah denoising and segmentation model [MS89]. Also, notice that unlike the previous two models, it outputs two objects: the completed and cleaned image $u$, and its associated edge collection $\Gamma$. 
For a given edge layout $\Gamma$, variation of $J_{\mathrm{ms}}\left[u \mid \Gamma, u^{0}, D\right]$ gives

$$
\gamma \Delta u+\lambda_{D}(x)\left(u^{0}-u\right)=0 \quad \text { on } \Omega \backslash \Gamma,
$$

with the natural adiabatic condition $\partial u / \partial \vec{\nu}=0$ along both $\Gamma$ and $\partial \Omega$.

Denote the solution to the elliptic equation (9) by $u_{\Gamma}$. Then the steepest descent infinitesimal move of $\Gamma$ for $J_{\mathrm{ms}}\left[\Gamma \mid u_{\Gamma}, u^{0}, D\right]$ is given by

$$
\frac{d x}{d t}=\left(\alpha \kappa+\left[\frac{\gamma}{2}\left|\nabla u_{\Gamma}\right|^{2}+\frac{\lambda_{D}}{2}\left(u_{\Gamma}-u^{0}\right)^{2}\right]_{\Gamma}\right) \vec{n} .
$$

Here $x \in \Gamma$ is an edge pixel and $\vec{n}$ the normal direction at $x$. The symbol $[g]_{\Gamma}$ denotes the jump of a scalar field $g(x)$ across $\Gamma$ :

$$
[g]_{\Gamma}(x)=\lim _{\sigma \rightarrow 0^{+}}(g(x+\sigma \vec{n})-g(x-\sigma \vec{n})) .
$$

The sign of the curvature $\kappa$ and the direction of the normal $\vec{n}$ are coupled so that $\kappa \vec{n}$ points to curvature center of $\Gamma$ at $x$.

Note that the curve evolution equation (10) is a combination of the mean curvature motion [ES91]

$$
d x / d t=\alpha \kappa \vec{n}
$$

and a field-driven motion specified by the second term. The field-driven motion attracts the curve toward the expected edge set, while the mean curvature motion makes sure that the curve does not develop ripples and stays smooth.

Like the TV inpainting model, inpainting based on the Mumford-Shah image model is of second order. But the extra complexity comes from its free boundary nature. In [CS01a, TAYW01], the level-set method of Osher and Sethian [OS88] is proposed.

In the most recent work by Esedoglu and Shen [ES02], a simpler numerical scheme is developed based on the $\Gamma$-convergence theory of Ambrosio and Tortorelli [AT90, AT92].

In the $\Gamma$-convergence theory, the 1 -dimensional edge $\Gamma$ is approximately represented by its associated signature function

$$
z: \Omega \rightarrow[0,1]
$$

which is nearly 1 almost everywhere except on a narrow (specified by a small parameter $\epsilon$ ) tubular neighborhood of $\Gamma$, where it is close to 0 . The posterior energy $J_{\mathrm{ms}}\left[u, \Gamma \mid u^{0}, D\right]$ is approximated by:

$$
\begin{aligned}
J_{\epsilon}\left[u, z \mid u^{0}, D\right]= & \frac{1}{2} \int_{\Omega} \lambda_{D}(x)\left(u-u^{0}\right)^{2} d x+\frac{\gamma}{2} \int_{\Omega} z^{2}|\nabla u|^{2} d x \\
& +\alpha \int_{\Omega}\left(\epsilon|\nabla z|^{2}+\frac{(1-z)^{2}}{4 \epsilon}\right) d x .
\end{aligned}
$$


Taking variation on $u$ and $z$ separately yields the Euler-Lagrange system:

$$
\begin{aligned}
\lambda_{D}(x)\left(u-u^{0}\right)-\gamma \nabla \cdot\left(z^{2} \nabla u\right) & =0 \\
\left(\gamma|\nabla u|^{2}\right) z+\alpha\left(-2 \epsilon \Delta z+\frac{z-1}{2 \epsilon}\right) & =0,
\end{aligned}
$$

with the natural adiabatic boundary conditions along $\partial \Omega$ (due to the boundary integrals coming from integration-by-parts):

$$
\frac{\partial u}{\partial \vec{\nu}}=0, \quad \frac{\partial z}{\partial \vec{\nu}}=0
$$

Define two elliptic operators acting on $u$ and $z$ separately:

$$
\begin{aligned}
L_{z} & =-\nabla \cdot z^{2} \nabla+\lambda_{D} / \gamma \\
M_{u} & =\left(1+2(\epsilon \gamma / \alpha)|\nabla u|^{2}\right)-4 \epsilon^{2} \Delta .
\end{aligned}
$$

Then the Euler-Lagrange system (12) and (13) is simply written as

$$
L_{z} u=\left(\lambda_{D} / \gamma\right) u^{0} \quad \text { and } \quad M_{u} z=1 .
$$

This coupled system can be solved easily by any efficient elliptic solver and an iterative scheme. Two digital examples are included in Figure 5 and 6.
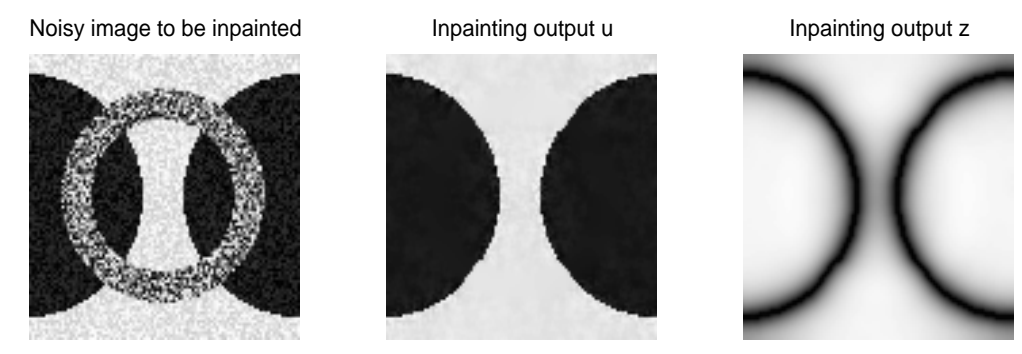

Figure 5. Inpainting based on the $\Gamma$-convergence approximation (11) and its associated elliptic system (16).

\subsection{Inpainting via Mumford-Shah-Euler image model}

Like the TV image model, the Mumford-Shah image model is insufficient for large-scale image inpainting problems due to the embedded length curve energy. To improve, Esedoglu and Shen [ES02] recently proposed the inpainting scheme based on the Mumford-Shah-Euler image model. 

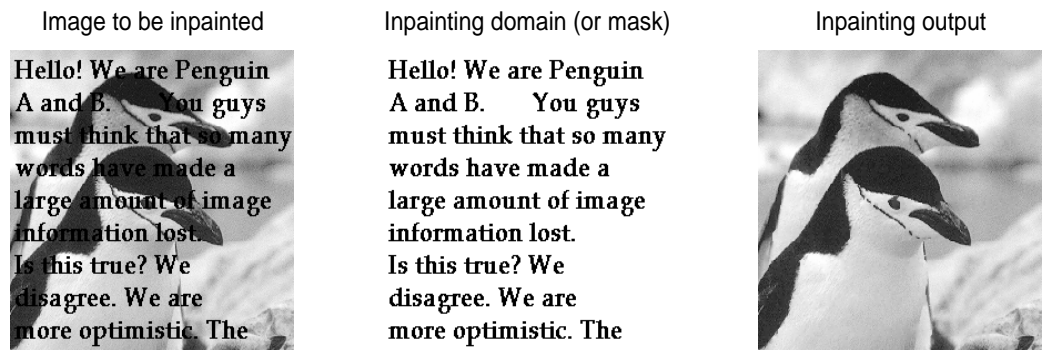

Figure 6. Text erasing by inpainting based on the Mumford-Shah image model.

In this model, the posterior energy to be minimized is

$J_{\mathrm{mse}}\left[u, \Gamma \mid u^{0}, D\right]=\frac{\gamma}{2} \int_{\Omega \backslash \Gamma}|\nabla u|^{2} d x+\int_{\Gamma}\left(\alpha+\beta \kappa^{2}\right) d s+\frac{\lambda}{2} \int_{\Omega \backslash D}\left(u-u^{0}\right)^{2} d x$,

where the length energy in $J_{\mathrm{ms}}$ has been upgraded to Euler's elastica energy.

As in the previous inpainting model, for a given edge layout $\Gamma$, the EulerLagrange equation for $J_{\mathrm{mse}}\left[u \mid \Gamma, u^{0}, D\right]$ is

$$
\gamma \Delta u+\lambda_{D}(x)\left(u^{0}-u\right)=0, \quad x \in \Omega \backslash \Gamma,
$$

with the adiabatic condition along $\Gamma$ and $\partial \Omega$ : $\partial u / \partial \vec{\nu}=0$.

For the solution $u_{\Gamma}$ to this equation, the infinitesimal steepest descent move of $\Gamma$ is given by [CKS01, Mum94a, LS84]:

$$
\frac{d x}{d t}=\alpha \kappa-\beta\left(2 \frac{d^{2} \kappa}{d s^{2}}+\kappa^{3}\right)+\left[\frac{\gamma}{2}\left|\nabla u_{\Gamma}\right|^{2}+\frac{\lambda_{D}}{2}\left(u_{\Gamma}-u^{0}\right)^{2}\right]_{\Gamma} .
$$

The meaning of the symbols is the same as in the previous section.

The digital implementation of this 4th order nonlinear evolutionary equation is highly non-trivial. The challenge lies in finding an effective numerical representation of the 1-dimensional object $\Gamma$, and robust ways to compute its geometry, i.e., the curvature and its differentials.

In Esedoglu and Shen [ES02], the equation is numerically implemented based on the $\Gamma$-convergence theory of De Giorgi [Gio61]. As for the previous Mumford-Shah image model, $\Gamma$-convergence approximation leads to simple elliptic systems that can be solved efficiently in computation.

De Giorgi [Gio61] proposed to approximate Euler's elastica curve model

$$
e(\Gamma)=\int_{\Gamma}\left(\alpha+\beta \kappa^{2}\right) d s
$$


by an elliptic integral of the signature $z$ (the two constants $\alpha$ and $\beta$ may vary):

$$
E_{\epsilon}[z]=\alpha \int_{\Omega}\left(\epsilon|\nabla z|^{2}+\frac{W(z)}{4 \epsilon}\right) d x+\frac{\beta}{\epsilon} \int_{\Omega}\left(2 \epsilon \Delta z-\frac{W^{\prime}(z)}{4 \epsilon}\right)^{2} d x
$$

where $W(z)$ can be the symmetric double-well function

$$
W(z)=\left(1-z^{2}\right)^{2}=(z+1)^{2}(z-1)^{2} .
$$

Unlike the choice of $W(z)=(1-z)^{2}$ for the Mumford-Shah image model, here the edge layout $\Gamma$ is embedded as the zero level-set of $z$. Asymptotically, as $\epsilon \rightarrow 0^{+}$, a boundary layer grows to realize the sharp transition between the two well states $z=1$ and $z=-1$.

Then the original posterior energy $J_{\mathrm{mse}}$ on $u$ and $\Gamma$ can be replaced by an elliptic energy on $u$ and $z$ :

$$
J_{\epsilon}\left[u, z \mid u^{0}, D\right]=\frac{\gamma}{2} \int_{\Omega} z^{2}|\nabla u|^{2} d x+E_{\epsilon}[z]+\frac{1}{2} \int_{\Omega} \lambda_{D}\left(u-u^{0}\right)^{2} d x .
$$

For a given edge signature $z$, variation on $u$ in $J_{\epsilon}\left[u \mid z, u^{0}, D\right]$ gives

$$
\lambda_{D}\left(u-u^{0}\right)-\gamma \nabla \cdot\left(z^{2} \nabla u\right)=0,
$$

with the adiabatic boundary condition $\partial u / \partial \vec{\nu}=0$ along $\partial \Omega$. For the solution $u$, the steepest decent marching of $z$ for $J_{\epsilon}\left[z \mid u, u^{0}, D\right]$ is given by

$$
\begin{aligned}
\frac{\partial z}{\partial t} & =-\gamma|\nabla u|^{2} z+\alpha g+\frac{\beta W^{\prime \prime}(z)}{2 \epsilon^{2}} g-4 \beta \Delta g, \\
g & =2 \epsilon \Delta z-\frac{W^{\prime}(z)}{4 \epsilon}
\end{aligned}
$$

again with the Neumann adiabatic conditions along the boundary $\partial \Omega$ :

$$
\frac{\partial z}{\partial \vec{\nu}}=0, \quad \text { and } \quad \frac{\partial g}{\partial \vec{\nu}}=0 .
$$

Eq. (24) is of fourth-order for $z$, with the leading head $-8 \epsilon \beta \Delta^{2} z$. Thus, to ensure stability, an explicit marching scheme would require $\Delta t=O\left((\Delta x)^{4} / \epsilon \beta\right)$. There are a couple of ways to stably increase the marching size. First, as inspired by Marquina and Osher [MO99], one can add a time correcting factor (as in Section 3.2):

$$
\frac{\partial z}{\partial t}=T(\nabla z, g \mid u)\left(-\gamma|\nabla u|^{2} z+\alpha g+\frac{\beta W^{\prime \prime}(z)}{2 \epsilon^{2}} g-4 \beta \Delta g\right),
$$

where $T(\nabla z, g, \mid u)$ is a suitable positive scalar, for example, $T=|\nabla z|$ [MO99]. 
The second alternative is to turn to implicit or semi-implicit schemes. Eq. (24) can be rearranged to

$$
\frac{\partial z}{\partial t}+\gamma|\nabla u|^{2} z-2 \alpha \epsilon \Delta z+8 \beta \epsilon \Delta^{2} z=-\frac{\alpha}{4 \epsilon} W^{\prime}(z)+\frac{\beta W^{\prime \prime}(z)}{2 \epsilon^{2}} g+\frac{\beta}{\epsilon} \Delta W^{\prime}(z),
$$

Or simply

$$
\frac{\partial z}{\partial t}+L_{u} z=f(z)
$$

where $L_{u}$ denotes the positive definite elliptic operator ( $u$-dependent)

$$
L_{u}=\gamma|\nabla u|^{2}-2 \alpha \epsilon \Delta+8 \beta \epsilon \Delta^{2},
$$

and $f(z)$ the entire right hand side of (26). Then a semi-implicit scheme can be designed as: at each discrete time step $n$,

$$
\left(1+\Delta t L_{u}\right) z^{(n+1)}=z^{(n)}+\Delta t f\left(z^{(n)}\right)
$$

where the positive definite operator $1+\Delta t L_{u}$ is numerically inverted based on many fast solvers [GO92, Str93]. A digital example is given in Figure 7.
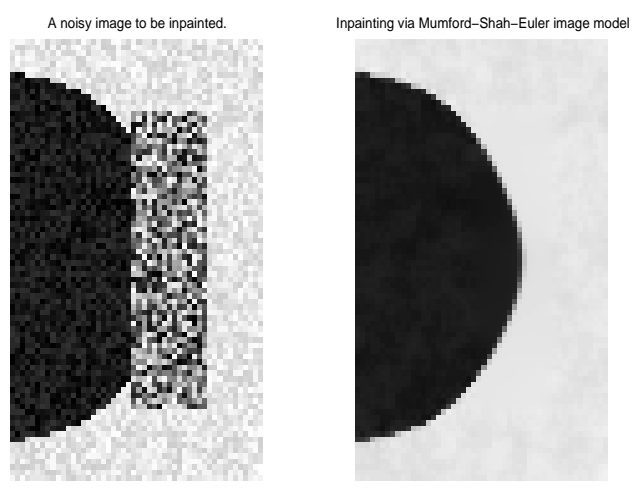

Figure 7. Inpainting based on the Mumford-Shah-Euler image model can satisfactorily restore a smooth edge as expected.

\section{Conclusion and open problems}

In this paper, we have surveyed all the recent inpainting models based on the combination of the Bayesian principle and geometric image models. As for the classical denoising, deblurring, and segmentation applications, the Bayesian framework has proven to be very effective in designing and improving general inpainting models. 
We have explained that the fundamental ingredient for a geometric image model is the associated or embedded curve model. Based on some natural axioms such as the Euclidean invariance and reversal invariance, we have been able to understand the general structure of geometric curve model on the 2dimensional image domain. We have described two general ways for "lifting" a curve model to a geometric image model.

We have observed that conventional first-order geometric image models, such as the TV model and Mumford-Shah model, function very well for classical denoising, deblurring, and segmentation problems, as well as for inpainting problems with a more local nature (such as zoom-in and text erasing). But for large-scale inpainting problems, they are insufficient for reconstructing perceptually meaningful outputs. Therefore, high order geometric image models, such as the elastica model and the Mumford-Shah-Euler model, become necessary for more general inpainting applications. The tradeoff is that high order inpainting models are computationally much more challenging.

We have described all the Euler-Lagrange PDE's associated to these models, their geometric meaning, and their digital realization based on techniques from numerical PDE's and the $\Gamma$-convergence theory.

Finally we post three interesting open problems.

(i) Video inpainting. Video inpainting has profound application in the movie industry, surveillance analysis, and dynamic vision analysis. The first open problem is: how to integrate the extra dimension of "time" into the spatial inpainting techniques? And how to define geometric prior models for spatial-temporal images?

(ii) Texture inpainting. Textures by definition are image patterns with rich statistical features. Geometric image models can well describe the boundaries of different texture patches, but are apparently insufficient for inpainting the textures themselves. Therefore, the second open problem is: how to integrate geometric image models and statistical texture models? And how to grow textures through texture synthesis without creating artificial boundaries?

(iii) Fast and efficient digital realization. Throughout this survey, numerical PDE has been a core computational tool for all the geometric inpainting models. The third open problem concerns fast and efficient digital implementation of the associated PDE's, especially for the high order ones. There are a number of non-trivial questions that wait to be answered: How to develop discretization schemes that respect geometry, the curvature and its differentials, for examples? How to speed up convergence based on various numerical techniques such as the multigrid method and the multiresolution decomposition? since speed is always highly concerned in applications. Finally, the energies of high order inpainting 
models, such as the elastica and the Mumford-Shah-Euler models, often have many local energy wells. It is thus another important issue to develop numerical schemes that can efficiently avoid being trapped in local energy wells, as in Molecular Dynamics [MW97].

\section{Acknowledgments}

We would like to thank Professor Sapiro's group for first introducing us to the topic. We would like to acknowledge the support from both the Institute of Mathematics and its Applications (IMA) at University of Minnesota and the Institute of Pure and Applied Mathematics (IPAM) at UCLA during this project. We are very grateful for the help from Professors Stan Osher, Luminita Vese, Sang Ha Kang, Li-Tien Cheng, Jean-Michel Morel and Simon Masnou, Willard Miller, Peter Olver, Fadil Santosa, Bob Gulliver, Dan Kersten, Selim Esedoglu, and Rachid Deriche. 


\section{References}

[AKR97] S. Armstrong, A. Kokaram, and P.J.W. Rayner. Nonlinear interpolation of missing data using min-max functions. IEEE Int. Conf. Nonlinear Signal and Image Processings, 1997.

[AT90] L. Ambrosio and V. M. Tortorelli. Approximation of functionals depending on jumps by elliptic functionals via $\Gamma$-convergence. Comm. Pure Appl. Math., 43:999-1036, 1990.

[AT92] L. Ambrosio and V. M. Tortorelli. On the approximation of free discontinuity problems. Boll. Un. Mat. Ital., 6-B:105-123, 1992.

$\left[\mathrm{BBC}^{+} 01\right] \quad$ C. Ballester, M. Bertalmio, V. Caselles, G. Sapiro, and J. Verdera. Filling-in by joint interpolation of vector fields and grey levels. IEEE Trans. Image Process., 10(8):1200-1211, 2001.

[BBS01] M. Bertalmio, A. L. Bertozzi, and G. Sapiro. Navier-Stokes, fluid dynamics, and image and video inpainting. IMA Preprint 1772 at: www.ima.umn.edu/preprints/jun01, Juun, 2001.

[Bou00] M. Boutin. Numerically invariant signature curves. Int. J. Comp. Vision, 40(3):235-248, 2000.

[BSCB00] M. Bertalmio, G. Sapiro, V. Caselles, and C. Ballester. Image inpainting. Computer Graphics, SIGGRAPH 2000, July, 2000.

[CCBT01] L. Chanas, J. P. Cocquerez, and J. Blanc-Talon. Highly degraded sequences restoration and inpainting. Preprint, 2001.

[CKS01] T. F. Chan, S.-H. Kang, and J. Shen. Euler's elastica and curvature based inpaintings. SIAM J. Appl. Math., submitted. Available at UCLA CAM Report 2001-12 at: www.math.ucla.edu/ rimagers, 2001.

[CMS98] V. Caselles, J.-M. Morel, and C. Sbert. An axiomatic approach to image interpolation. IEEE Trans. Image Processing, 7(3):376-386, 1998.

$\left[\mathrm{COS}^{+} 98\right] \quad$ E. Calabi, P. J. Olver, C. Shakiban, A. Tannenbaum, and S. Haker. Differential and numerically invariant signature curves applied to object recognition. Int. J. Comp. Vision, 26(2):107-135, 1998.

[COT96] E. Calabi, P. J. Olver, and A. Tannenbaum. Affine geometry, curve flows, and invariant numerical approximations. Adv. Math., 124(1):154-196, 1996. 
[CS01a] T. F. Chan and J. Shen. Mathematical models for local non-texture inpaintings. SIAM J. Appl. Math., 62(4), 2001.

[CS01b] T. F. Chan and J. Shen. Morphologically invariant PDE inpaintings. UCLA CAM Report 2001-15 at: www.math.ucla.edu/rimagers; submitted to IEEE Trans. Image Process., 2001.

[CS01c] T. F. Chan and J. Shen. Non-texture inpainting by curvature driven diffusions (CDD). J. Visual Comm. Image Rep., 12(4):436-449, 2001.

[CS01d] T. F. Chan and J. Shen. Variational restoration of non-flat image features: models and algorithms. SIAM J. Appl. Math., 61(4):1338-1361, 2001.

[Don00] D. L. Donoho. Curvelets. Invited talk at workshop on Wavelets, Statistics, and Image Processing, Geogia Inst. Tech., 1999; Invited talk at MSRI workshop on Mathematics of Imaging; 1999. Beamlets, Invited talk at IMA workshop on Image Analysis and Low Level Vision, 2000.

[EM76] G. Emile-Male. The Restorer's Handbook of Easel Painting. Van Nostrand Reinhold, New York, 1976.

[ES91] L. C. Evans and J. Spruck. Motion of level sets by mean curvature. J. Diff. Geom., 33(3):635-681, 1991.

[ES02] S. Esedoglu and J. Shen. Digital inpainting based on the Mumford-Shah-Euler image model. UCLA CAM Report 2001-24 at: www.math.ucla.edu/־imagers; European J. Appl. Math., to appear, 2002.

[Fre61] H. Freeman. On the encoding of arbitrary geometric configuration. IRE Transactions on Electronic Computers, EC-10(2):260-268, 1961.

[GG84] S. Geman and D. Geman. Stochastic relaxation, Gibbs distributions, and the Bayesian restoration of images. IEEE Trans. Pattern Anal. Machine Intell., 6:721-741, 1984.

[Gib02] W. Gibbs. Elementary Principles of Statistical Mechanics. Yale University Press, 1902.

[Gio61] E. De Giorgi. Frontiere orientate di misura minima. Sem. Mat. Scuola Norm. Sup. Pisa, 1960-61.

[Giu84] E. Giusti. Minimal Surfaces and Functions of Bounded Variation. Birkhäuser, Boston, 1984.

[GO92] G. H. Golub and J. M. Ortega. Scientific Computing and Differential Equations. Academic Press, 1992.

[IP97] H. Igehy and L. Pereira. Image replacement through texture synthesis. Proceedings of IEEE Int. Conf. Image Processing, 1997.

[JCL94] K.-H. Jung, J.-H. Chang, and C. W. Lee. Error concealment technique using data for block-based image coding. SPIE, 2308:1466-1477, 1994.

[Kan79] G. Kanizsa. Organization in Vision. Praeger, New York, 1979. 
[KMFR95a] A. C. Kokaram, R. D. Morris, W. J. Fitzgerald, and P. J. W. Rayner. Detection of missing data in image sequences. IEEE Trans. Image Process., 11(4):1496$1508,1995$.

[KMFR95b] A. C. Kokaram, R. D. Morris, W. J. Fitzgerald, and P. J. W. Rayner. Interpolation of missing data in image sequences. IEEE Trans. Image Process., 11(4):1509-1519, 1995.

[KR96] D. C. Knill and W. Richards. Perception as Bayesian Inference. Cambridge Univ. Press, 1996.

[KS93] W. Kwok and H. Sun. Multidirectional interpolation for spatial error concealment. IEEE Trans. Consumer Electronics, 39(3), 1993.

[KS97] I. Karatzas and S. E. Shreve. Brownian motion and stochastic calculus. Springer, New York, 1997.

[LS84] J. Langer and D. A. Singer. The total squared curvature of closed curves. J. Diff. Geom., 20:1-22, 1984.

[MH80] D. Marr and E. Hildreth. Theory of edge detection. Proc. Royal Soc. London, B:207: 187-217, 1980.

[MM98] S. Masnou and J.-M. Morel. Level-lines based disocclusion. Proceedings of 5th IEEE Int'l Conf. on Image Process., Chicago, 3:259-263, 1998.

[MO99] A. Marquina and S. Osher. Lecture Notes in Computer Science, volume 1682, chapter "A new time dependent model based on level set motion for nonlinear deblurring and noise removal", pages 429-434. 1999.

[MS89] D. Mumford and J. Shah. Optimal approximations by piecewise smooth functions and associated variational problems. Comm. Pure Applied. Math., XLII:577-685, 1989.

[Mum94a] D. Mumford. Elastica and computer vision. In C. L. Bajaj, editor, Algebraic Geometry and its Applications, pages 491-506. Springer-Verlag, New York, 1994.

[Mum94b] D. Mumford. Geometry Driven Diffusion in Computer Vision, chapter "The Bayesian rationale for energy functionals", pages 141-153. Kluwer Academic, 1994.

[MW97] J. J. Moré and Z. Wu. Issues in large-scale global molecular optimization. In L. T. Biegler, T. F. Coleman, A. R. Conn, and F. N. Santosa, editors, LargeScale Optimization with Applications, pages 99-121. Springer, New York, 1997.

[NMS93] M. Nitzberg, D. Mumford, and T. Shiota. Filtering, Segmentation, and Depth. Lecture Notes in Comp. Sci., Vol. 662. Springer-Verlag, Berlin, 1993.

[OR90] S. Osher and L. Rudin. Feature-oriented image enhancement using shock filters. SIAM J. Num. Anal., 27(4):919-940, 1990. 
[OS88] S. Osher and J. A. Sethian. Fronts propagating with curvature-dependent speed: Algorithms based on Hamilton-Jacobi formulations. J. Comput. Phys., 79(12), 1988.

[PM90] P. Perona and J. Malik. Scale-space and edge detection using anisotropic diffusion. IEEE Trans. Pattern Anal. Machine Intell., 12:629-639, 1990.

[RO94] L. Rudin and S. Osher. Total variation based image restoration with free local constraints. Proc. 1st IEEE ICIP, 1:31-35, 1994.

[ROF92] L. Rudin, S. Osher, and E. Fatemi. Nonlinear total variation based noise removal algorithms. Physica D, 60:259-268, 1992.

[Str93] G. Strang. Introduction to Applied Mathematics. Wellesley-Cambridge Press, MA, 1993.

[TAYW01] A. Tsai, Jr. A. Yezzi, and A. S. Willsky. Curve evolution implementation of the Mumford-Shah functional for image segmentation, denoising, interpolation and magnification. IEEE Trans. Image Process., 10(8):1169-1186, 2001.

[Wal85] S. Walden. The Ravished Image. St. Martin's Press, New York, 1985.

[WL00] L.-Y. Wei and M. Levoy. Fast texture synthesis using tree-structured vector quantization. Preprint, Computer Science, Stanford University, 2000; Also in Proceedings of SIGGRAPH, 2000.

[ZM97] S. C. Zhu and D. Mumford. Prior learning and Gibbs reaction-diffusion. IEEE Trans. on PAMI, 19(11):1236-1250, 1997.

[ZWM97] S. C. Zhu, Y. N. Wu, and D. Mumford. Minimax entropy principle and its applications to texture modeling. Neural Computation, 9:1627-1660, 1997. 